\title{
When Systems Biology Meets Metabolomics: Network Models May Guide Cancer Therapy
}

\author{
Xin Wen, Wan Li and Bo Liu*
}

State Key Laboratory of Biotherapy and Cancer Center \& School of Life Sciences, Sichuan University, Chengdu 610041, China

\section{What is Metabolomics?}

Hitherto, significant biological progress has allowed a comprehensive investigation of "so-called" omics, including genomics, transcriptomics, proteomics and metabolomics, which may provide a better understanding of the underlying mechanisms in complex biological systems [1]. Compared to other omic sciences such as genomics, transcriptomics, and proteomics, metabolomics has existed for several decades but received no much attention. Metabolomics is well-known to be a technique involved in a global detection of many small molecule metabolites and identification of metabolic preprogramming (e.g., some changes occurring in metabolic pathways and phenotypes) [2]. Recent advances in mass spectrometry (MS), nuclear magnetic resonance (NMR), as well as, gas and liquid chromatography, has led to a trend for utilization of metabolomics in cancer therapy [3].

\section{Classical Metabolomic Approaches and Systems Biology}

Classical approaches to metabolomic studies mainly included NMR, which was favored by machine accessibility, established data handling and the non-destructive nature of analysis. But, NMR has been gradually complemented by MS because both can have more advantages. Of Note, MS provides higher sensitivity, improved metabolite discrimination, full coverage of metabolome space and can be characterized by modularity to perform compoundclass-specific analyses. Additionally, MS can be coupled to gas and liquid chromatography. However, these classical approach-based metabolomics studies have some limitations, such as the timing or temporal relationship of biological intermediates and variability across individuals. Therefore, systems biology, a combined computational and experimental approach to analyzing complex biological systems, has been emerging to enable the production of metabolic network models reconstructed from genome sequences, and experimental measurements of metabolome [4].

\section{Systematic Network Models Elucidate Metabolomics}

Complexity of the metabolomics network may inspire a series of systems biology approaches to uncover its complicated nature by using some mathematical models such as ordinary differential equations, Petri nets, Bayesian networks, Boolean model and etc. [5,6]. For instance, Naive Bayesian model is well-suited to integrate these high-throughput data from different types of biological evidence and make the final interaction predictions in an integrated way; thereby, constructing the metabolomics network. Following the Bayesian theorem, we compute the posterior odds given $\mathrm{n}$ evidence as follows:

$$
o_{\text {posterior }}=\frac{P\left(\text { positive } \mid E_{1}, \ldots, E_{n}\right)}{P\left(\text { negative } \mid E_{1}, \ldots, E_{n}\right)} \text { Where positive means that two }
$$

proteins are functional related while negative means not. We define

$$
L R_{\left(E_{1}, \ldots, E_{n}\right)}=\frac{P\left(E_{1}, \ldots, E_{n} \mid \text { positive }\right)}{P\left(E_{1}, \ldots, E_{n} \mid \text { negative }\right)} \text { Then }
$$

Oposterior = $O_{\text {prior }}^{\star} L R$. As Naive Bayesian model supposes that each of the evidence is conditional independent, we can simplify LR as

$$
L R_{\left(E_{1}, \ldots, E_{n}\right)}=\prod_{i=1}^{n} L R_{\left(E_{i}\right)}
$$

As the prior odd is a constant, the composite LR corresponding to a type of specific biological evidence can be used to measure the predictive power or confidence degree for predicting links.

\section{Systems Biology and Metabolomics in Cancer Therapy}

Emerging therapeutic strategies have been considered to mediate cancer cell death by regulating oncogene or tumor suppressor-related signaling pathways, as well as to remodel the structure of metabolomics network [7]. A comprehensive knowledge of metabolomics network may provide a basic framework for uncovering the biology of cancer as an integrated system [8]. With increasing metabolomics-wide data, robust mathematical models (i.e., Naïve Bayesian network is wellsuited for integrating disparate types of data) seem to be imperative for utilization of metabolomics in cancer therapy [9]. That is to say, traditional ways of studying metabolomics can be complemented by systems biology approaches in current and future cancer therapeutics.

\section{References}

1. Ram PT, Mendelsohn J, Mills GB (2012) Bioinformatics and systems biology Mol Oncol 6: 147-154

2. Wishart DS (2008) Applications of metabolomics in drug discovery and development. Drugs R D 9: 307-322.

3. Kell DB (2006) Systems biology, metabolic modelling and metabolomics in drug discovery and development. Drug Discov Today 11: 1085-1092.

4. Kreeger PK, Lauffenburger DA (2010) Cancer systems biology: a network modeling perspective. Carcinogenesis 31: 2-8.

5. Barabasi AL, Oltvai ZN (2004) Network biology: understanding the cell's functional organization. Nat Rev Genet 5: 101-113.

6. Lavrik IN (2010) Systems biology of apoptosis signaling networks. Curr Opin Biotechnol 21: 551-555.

7. Kreeger PK, Lauffenburger DA (2010) Cancer systems biology: a network modeling perspective. Carcinogenesis 31: 2-8.

8. Cui Q, Ma Y, Jaramillo M, Bari H, Awan A, et al. (2007) A map of human cancer signaling. Mol Syst Biol 3: 152.

9. D'Alessandro A, Zolla L (2012) Metabolomics and cancer drug discovery: let the cells do the talking. Drug Discov Today 17: 3-9.

*Corresponding author: Bo Liu, State Key Laboratory of Biotherapy and Cancer Center \& School of Life Sciences, Sichuan University, Chengdu 610041, China, Tel./Fax: +86 28 85164063; E-mail: liubo2400@163.com

Received August 24, 2012; Accepted August 25, 2012; Published August 27 , 2012

Citation: Wen X, Li W, Liu B (2012) When Systems Biology Meets Metabolomics: Network Models May Guide Cancer Therapy. Metabolomics 2:e116. doi:10.4172/2153-0769.1000e116

Copyright: (c) 2012 Wen X, et al. This is an open-access article distributed under the terms of the Creative Commons Attribution License, which permits unrestricted use, distribution, and reproduction in any medium, provided the original author and source are credited. 